\title{
Observational Implications of Cosmological Event Horizons
}

\author{
Nemanja Kaloper*† \\ UC Davis \\ E-mail: kaloper@physics.ucdavis.edu
}

\begin{abstract}
In an eternally accelerating universe observers are surrounded by event horizons. The horizons restrict the observer's access to the information about the universe. This implies a bound $N \sim 60$ on the number of e-folds of inflation which we can observe if the scale of the dark energy today is $\sim\left(10^{-3} \mathrm{eV}\right)^{4}$ and its equation of state forever remains $w<-1 / 3$. The bound is independent of the duration of inflation, or the duration of out scrutiny of the sky. It arises because the imprints of the inflationary perturbations "inflate away" just like the initial inhomogeneities during ordinary inflation. The current CMB data may be giving us as much information about the history of the universe as we can ever hope to get.
\end{abstract}

28th Johns Hopkins Workshop on Current Problems in Particle Theory

June 5-8, 2004

Johns Hopkins University Homewood campus - Bloomberg Center for Physics and Astronomy, Baltimore, Maryland

\footnotetext{
* Speaker.

${ }^{\dagger}$ This work was supported in part by the DOE Grant DE-FG03-91ER40674, in part by the NSF Grant PHY-0332258 and in part by a Research Innovation Award from the Research Corporation.
} 


\section{Introduction}

Observations indicate that the universe may be accelerating [1, 2, 3]. If so the universe is dominated by a dark energy component with equation of state $w=p / \rho \lesssim-2 / 3$, comprising as much as $70 \%$ of the critical energy density, $\rho_{c} \sim\left(10^{-3} \mathrm{eV}\right)^{4}$. Usually dark energy is modelled either by a cosmological constant or a quintessence field [4, 5]. If the equation of state of dark energy obeys $w<-1 / 3$ forever, the universe will continue to accelerate indefinitely. In such case any observer is surrounded by an event horizon, which limits how much of the universe she can see [6, 7, 8, 9]. Recently there has been discussion about the issue of compatibility between late eternal acceleration and long early inflation, motivated by the interpretational questions about holography in de Sitter space [10, 11, 12]. If one adopts the covariant entropy bounds [13, 14] as a measure of entropy in cosmology, then the late acceleration does not yield a bound on the duration of early inflation, but does produce a bound on how much of the early inflation will ever be observable [12]. If our universe accelerates forever, we will never see past the last 60 e-folds or so.

We have also found that a universe which accelerates eternally in the future contains the most information about the early inflationary perturbations at the epoch of transition to the late acceleration [12]. After this time, observers will be able to observe less and less about the inflationary phase. This is because the fluctuations generated during inflation cease to come back into the horizon, and those that did return will be evicted again. The overall amplitude of the CMB will redshift, and more significantly the pattern of anisotropies will freeze in such a way that little new information will become available. Eventually, the CMB will redshift to a point where it is permanently contaminated by cosmological Hawking radiation. This is the cosmological analogue of the "frozen star" picture in black hole physics, corresponding to the indefinite reddening of the surface of a collapsing cloud of matter.

If the universe does not stop to accelerate, the current observations may be already providing us with all the information about the early universe that we can ever hope to get. This leads to an interesting new twist on the "Why Now?" problem:

Why is now ( \pm few current Hubble times) the best time to observe the signatures of early universe physics?

In this note we will review these issues.

\section{E-fold Counts and Holography}

We adopt the point of view that in backgrounds with horizons, the entropy in the spacetime conforms to the covariant entropy bounds of [13, 14]. Then the existence of a horizon constrains only the entropy inside the final Hubble volume to not exceed the area of its horizon. This bound cannot restrict the entropy deposited outside ${ }^{1}$ of it. The covariant bound is clearly not violated in our universe, as the entropy of the observable universe is many orders of magnitude below the horizon area. To an observer inside the causal patch in Fig. 1, the portion of the reheating surface RS which is outside the event horizon lies beyond her causal future, in a sense. Hence she can't

\footnotetext{
${ }^{1}$ However, it is still very much unclear how to think about the entropy deposited in the region outside of the horizon the viewpoint of any given observer.
} 
ever see that inflation ended there, and that any entropy has been released, or for that matter, that inflation even happened there.

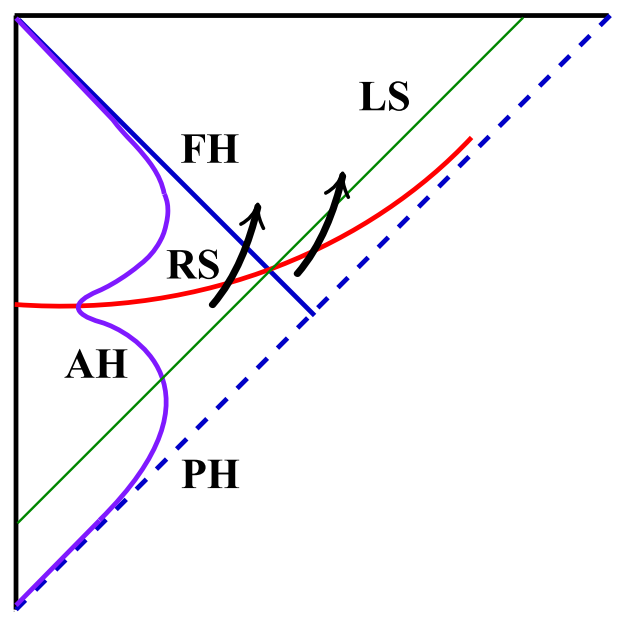

Figure 1: Causal patch of an observer in a universe where inflation and reheating are followed by eternal accelerated expansion. The symbols designate: $\mathrm{PH}$ and $\mathrm{FH}$ - past and future sections of the event horizon, $A H$ - apparent, or Hubble, horizon, RS - reheating surface, and LS - a future oriented light sheet, which intersects both the event horizon and the infinitely inflated future. Black arrows are the worldlines of the entropy released at the end of inflation.

Thus outside of the event horizon we constrain the entropy on the portion of the reheating surface in that region using the lightsheet labelled LS in Fig. 1. According to the covariant bound [14], the entropy that crosses any segment of a lightsheet is bounded by a maximum of the area along this segment, in Planck units. Because LS intersects the infinitely inflated future at the top of the diagram, and so its maximal area diverges, the covariant entropy bound does not give an interesting constraint. It can easily accommodate the entropy released after arbitrarily long inflation. For a more detailed discussion of these issues, see [11, 12]. Similar difficulties with adding up entropy on large, arbitrarily chosen spacelike surfaces have been noticed earlier in [13, 14, 15]. They are consistently resolved by the application of the covariant bound.

\section{How Far Can We See?}

Given that horizons do not restrict the duration of inflation in the early universe, we can ask if they restrict how much of it we can see. We will now review the argument of [12], that in the spacetimes undergoing eternal acceleration the event horizon limits the total number of e-folds that we can ever observe to the last $N \sim 60$ or so.

We study inflation by observing temperature and density contrasts on the sky, that were produced earlier in inflation. To solve the horizon and flatness problems, inflation must have lasted at least $N \sim 60$ e-folds [16]. During this stage quantum fluctuations are imprinted on the curvature and are subsequently stretched by inflation to super (Hubble) horizon scales [17, 18], where they "freeze out": their amplitude approaches a constant set by the horizon crossing condition, whereas their wavelength scales with the particle horizon, $\lambda(t)=\lambda_{0} a(t) / a_{0}$. Their future evolution depends 
on the subsequent evolution of the universe. If inflation ends and reheating occurs, the Hubble horizon will grow linearly in time, while the wavelength stretches more slowly, as $\lambda \sim a(t)$. If after inflation the vacuum energy is zero, after a long enough time the Hubble horizon catches up with any perturbation, of any given initial wavelength (see the left panel of Fig. 2). The perturbation will reenter the horizon and "melt": it will oscillate and seed structure formation via the Jeans instability [17, 18]. Hence in such case a patient observer would be able to see arbitrarily far back
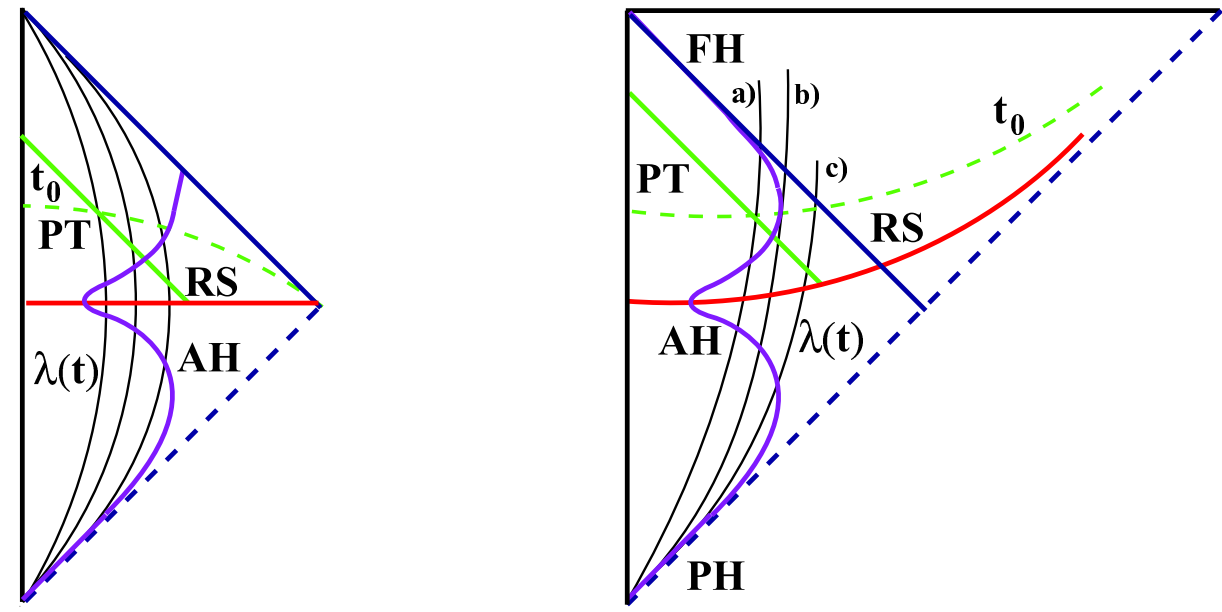

Figure 2: Evolution of the wavelengths of some typical inflationary perturbations in the causal patch in a universe without (left panel) and with (right panel) event horizons. In the left panel, all fluctuations eventually reenter the Hubble horizon. In the right panel, in the case $a$ ), a fluctuation is stretched outside of the Hubble horizon during inflation, remains there for a time, then reenters during a matter dominated era after inflation, and eventually gets expelled out of the horizon once more during the final stage of acceleration. In the case $b$ ), the fluctuation could have reentered about now, but the late acceleration pushes it back out. In the case c), the late acceleration prevents the fluctuation from ever reentering the Hubble horizon.

into inflation. The longer she waits, the earlier the fluctuations she sees were created.

On the other hand, if a small positive vacuum energy remains after inflation, the universe will start to accelerate again after some time, continuing to do so forever. There will be event horizon as in the right panel of Fig. 2, rendering a huge part of the global spacetime permanently inaccessible to any given observer. In this case, the inflationary fluctuations could either (see the right panel of Fig. 2): a) reenter the Hubble horizon during matter domination, and then be expelled again in the far future, $b$ ) in the marginal case, have a wavelength which equals the Hubble horizon size at about the time when the universe begins to accelerate again, or c) never reenter, remaining instead outside the Hubble horizon forever after their eviction from it during early inflation.

An observer learns about inflation by examining the structures which form by the accretion of matter in the gravitational wells formed from the perturbations which may reenter the horizon, by observing anisotropies induced by these wells in the temperature of the thermal photons emitted at reheating. ${ }^{2}$ The observer in an accelerating universe will notice a gradual loss of the information about inflation in the course of time. She will notice fewer new structures at the largest scales, because the inflationary fluctuations cease to enter the horizon after the onset of late acceleration.

\footnotetext{
${ }^{2}$ We ignore the difference between the reheating surface and the last scattering surface here for the sake of simplicity.
} 
She will also notice the breakup of the structures that have already begun forming, as they are torn up by the late acceleration. In due course, all the inflationary fluctuations which re-entered during radiation and matter domination will be pushed back out of the Hubble horizon, whose interior will be smoothed out again, at least on large scales.

The CMB photons originate on the slice (i.e. a sphere) of the last scattering surface (RS), separated from the observer by null geodesics (labelled PT, for photon trajectory, in Fig. 2.). The inflationary fluctuations are imprinted on them en route to the observer, appearing as a distribution of hot and cold spots on the last scattering surface. In a decelerating universe, the radius of this last scattering sphere grows without bound, the pattern of spots changes, and new information about inflation continues to become available over time. Eventually, if one continued to observe the pattern of anisotropies in the $\mathrm{CMB}$, the entire history of the inflationary period would (in principle) be available.

If a universe accelerates at late times, the last-scattering sphere asymptotes to the size of the event horizon at the time of last scattering, which is finite. In this case waiting a given period of time will correspond to a smaller and smaller change in the size of the last-scattering sphere. The size converges to a constant (or changes extremely slowly, in the case of quintessence). The pattern of anisotropies in the CMB will "freeze" after the transition to future acceleration, first on the largest scales, and then on shorter and shorter scales. Therefore observations made after the beginning of the late acceleration will not reveal any information about periods of inflation earlier than those that have already been seen, and will at best slightly improve the data on the already visible period. The acceleration freezes an ever-fainter image of one slice of the last scattering surface on the sky, for a very long time. This is the cosmological analogue of the "frozen star" concept in black hole physics.

Even this information will be erased. Spacetimes with event horizons contain Hawking particles. As the cosmological expansion advances, the $\mathrm{CMB}$ will cool until the number of $\mathrm{CMB}$ photons counted by an observer drops below the number of Hawking photons. After that, the information in the CMB will be masked by the "noise" in the cosmological Hawking radiation. Asymptotically the bath of Hawking particles will completely overwhelm the CMB. Some implications of the loss of a record of the last stages of inflation for astronomy have been discussed in [19. 20, 21].

We now turn to the quantitative statement of the bound. For inflation to solve the horizon and flatness problems, it must yield $N$ e-folds which will expand the initial Hubble patch into a region at least of the size of the present Hubble horizon size, $\left(H_{0}\right)^{-1} \sim\left(10^{-33} \mathrm{eV}\right)^{-1}$. The wavelengths of perturbations grow in time according to

$$
\lambda(t)=\lambda_{0} \frac{a(t)}{a\left(t_{0}\right)} .
$$

Taking $t_{0}$ to be $O$ (today) $\left(t_{0} \sim 10^{10}\right.$ years), we are interested in the largest scale observable now ${ }^{3}$, $\lambda_{0}=1 / H_{0}$. Such a perturbation originated during inflation at some time $t_{b}<t_{0}$, when its wavelength was the inflationary Hubble size, $\lambda\left(t_{b}\right)=1 / H\left(t_{b}\right)$. Hence,

$$
a\left(t_{b}\right) H\left(t_{b}\right)=a_{0} H_{0} .
$$

\footnotetext{
${ }^{3}$ We denote quantities evaluated at time $t=t_{0}$ with a subscript 0 .
} 
This equation is just the usual horizon crossing condition [18]. Approximating the inflating phase as de Sitter space with a constant Hubble scale $H_{i}$ and using the flat slicing, we get $a(t)=a_{e} \exp \left(H_{i}(t-\right.$ $\left.t_{e}\right)$ ) during inflation, where the subscript $e$ refers to the end of inflation. Evaluating this at a time $t_{b}$ during inflation and substituting into (3.2) yields

$$
N \equiv H_{i}\left(t_{e}-t_{b}\right)=\ln \left(\frac{a_{e} H_{i}}{a_{0} H_{0}}\right) .
$$

After inflation, the universe grew by a factor of about $a_{0} / a_{e} \sim T_{e} / T_{0}$, where $T_{e}$ is the reheating temperature and $T_{0} \sim 10^{-3} \mathrm{eV}$ the current CMB temperature. Taking $a_{0} / a_{e} \sim 10^{26}-10^{28}$ and assuming that the scale of inflation is $H_{i} \lesssim 10^{14} \mathrm{GeV}$, one finds $N \sim 60$. This may be sensitive to the reheating temperature, the scale of inflation etc, but we will ignore such details here (see [16]).
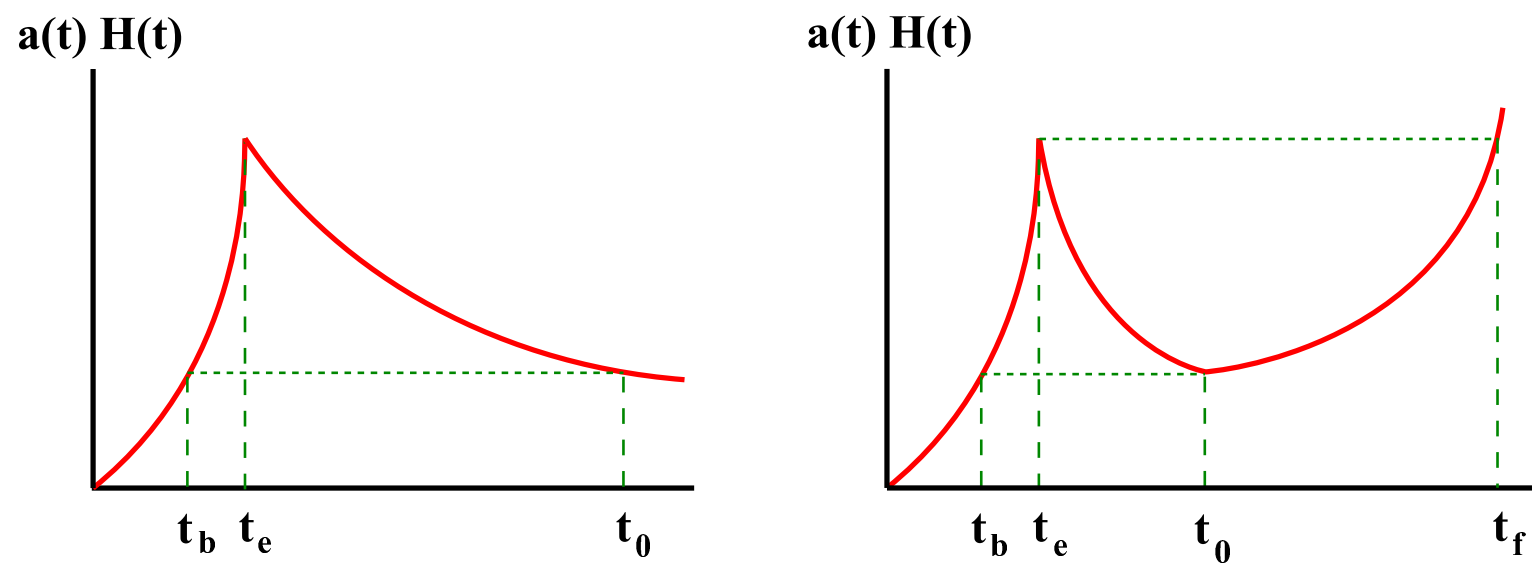

Figure 3: On the left, evolution of the comoving Hubble scale $a(t) H(t)$ for a universe which inflates, followed by radiation and matter domination; on the right, the same graph for a universe that enters a latetime accelerating phase.

To illustrate this equation, we plot the comoving Hubble scale $a(t) H(t)$ for a universe without any late epoch of cosmic acceleration in the left panel of Fig. 3. Initially, it grows exponentially because of inflation, but then it decreases as a small negative power of $t$, because after reheating the universe decelerates; e.g. if the universe is dominated by matter with an equation of state $p=w \rho$, $a(t) H(t)$ scales as $t^{-(1+3 w) /[3(1+w)]}$, which is decreasing for $w>-1 / 3$. As $a(t) H(t)$ decreases, it scans through more and more values of the comoving momentum $k=1 / \lambda_{0}$. Those are the scales that reenter the horizon. We see that regardless of how large a scale $\lambda_{0}$ is, if the universe decelerates forever and $a(t) H(t)$ continues to decrease, at some time this scale will reenter the Hubble horizon.

In contrast, if the universe accelerates in the future, the comoving Hubble scale $a(t) H(t)$ will grow again at late times, as we can see by setting $w<-1 / 3$ in the scaling law given above. At a time $t_{f}$, where $f$ stands for final, when the comoving Hubble scale equals its value at reheating, the very last perturbation generated during inflation will be pushed back out of the horizon. After $t_{f}$ no inflationary perturbations will remain in the Hubble horizon and no new structure will form from the seeds generated by inflation ${ }^{4}$ (see the right panel of Fig. 3 .). The time $t_{f}$ is defined by the

\footnotetext{
${ }^{4}$ In reality, the information about the primordial inflation encoded in the shortest scales generated during inflation will already be strongly contaminated by the nonlinear effects occurring in the intervening period between $t_{e}$ and $t_{f}$, such as galaxies, clusters etc. We are ignoring this contamination here.
} 
equality

$$
a\left(t_{f}\right) H\left(t_{f}\right)=a\left(t_{e}\right) H_{i},
$$

where $t_{e}$ is the time at reheating. The value of $t_{f}$ depends on the equation of state of the dark energy in a way we calculate below.

Moreover, spacetimes with event horizons contain Hawking particles, characterized by the Gibbons-Hawking temperature ${ }^{5} T_{H}=H / 2 \pi$ [22]. This temperature does not redshift in the usual way, because the Hawking radiation is continuously replenished by quantum fluctuations. Thus in an accelerating universe the CMB temperature $T_{C M B}$ will eventually redshift down to $T_{H} \sim H(t)$. If $T_{e}$ was the reheating temperature, under adiabatic evolution the temperature at any later time is related to it by $T_{C M B}(t)=T_{e} a\left(t_{e}\right) / a(t)$, and so the equality of CMB and Hawking radiation temperatures, $T_{C M B}\left(t_{T}\right)=T_{H}$, will occur at a time $t_{T}$ roughly given by

$$
a\left(t_{T}\right) H\left(t_{T}\right)=a\left(t_{e}\right) T_{e} .
$$

If reheating were perfectly efficient, the reheating temperature would be related to the Hubble scale at the end of inflation by $T_{e} \sim \sqrt{H_{i}}$ (recall that we have set the Planck mass equal to unity). Thus, since $H_{i}<1, T_{e}>H_{i}$, and $t_{T}>t_{f}$. In practice, however, the reheating temperature is model dependent ${ }^{6}$, and it is possible that there are some models where the ordering of $t_{f}$ and $t_{T}$ is reversed. But in this case, for $t>t_{T}$ the CMB photons will be outnumbered by Hawking photons, and it would be impossible to extract any information about inflation from their fluctuations.

If the late epoch of acceleration goes on forever, only those inflationary fluctuations with comoving momenta in the interval $a\left(t_{b}\right) H\left(t_{b}\right) \leq k \leq a\left(t_{e}\right) H\left(t_{e}\right)$ will ever be observable. Thus an observer will not see much past the last 60 e-folds of inflation, however patient she may be. Further, this information will be lost after the time $t_{T}$, the value of which depends on the equation of state of the dark energy $\left(t_{T} \sim t_{f}\right)$. It can be found by solving (3.5), eqs. (3.2) and (3.3), and the scaling $a(t) H(t) \sim a_{0} H_{0}\left(t / t_{0}\right)^{-(1+3 w) /[3(1+w)]}$ when $-1<w<-1 / 3$ :

$$
t_{T} \sim 10^{78(1+w) /|1+3 w|} t_{0} .
$$

When $w \rightarrow-1 / 3$ the time diverges, as expected since for $w \geq-1 / 3$ the event horizon and the Hawking particles disappear, and the information about early inflation survives and remains available to an investigation by a patient observer. The limit $w \rightarrow-1$ is simpler to determine by directly substituting $a\left(t_{T}\right) H\left(t_{T}\right)=a_{0} H_{0} \exp \left(H_{0} t_{T}\right)$, which yields

$$
t_{T} \sim \frac{60}{H_{0}} .
$$

Specifically, if the dark energy is a small cosmological constant, the record of early inflation will be lost in about a trillion years (see also [19, 20, 21] for astronomical implications of these time scales).

\footnotetext{
${ }^{5}$ This is certainly correct for a positive cosmological constant $(w=-1)$. For quintessential universes, we believe there is a similar effect [7], but we are not aware of a precise calculation of it.

${ }^{6} \mathrm{As}$ a result, the right-hand side of the equation (3.5) should really read $a\left(t_{R}\right) T_{R}$, and this quantity may evolve slightly differently. However, the differences will all be model dependent and confined to short scales, and so we will ignore this here.
} 


\section{Summary}

Having assumed the covariant entropy bounds [13, 14] we find that eternal dark energy with $w<-1 / 3$ limits us to seeing only those inflationary perturbations which originated after the ones currently observable. It also gradually dilutes the information available in the currently observable perturbations. Hence we can re-formulate the "Why Now?" problem in a new way: Why are we living in the time at which we can see back to the earliest scales? More specifically, why would the number of e-folds required to solve the horizon problem and explain the observed large scale homogeneity, isotropy and flatness of the universe also be the maximum number of e-folds which we will ever be able to observe?

\section{Acknowledgements}

We thank M. Kleban and L. Sorbo for collaboration and A. Albrecht, T. Banks, W. Fischler, G. Holder, M. Kaplinghat, A. Linde, M. Sloth, L. Susskind, S. Thomas, and T. Tyson for useful discussions.

\section{References}

[1] A. G. Riess et al., Astron. J. 116 (1998) 1009; S. Perlmutter et al., Astrophys. J. 517 (1999) 565.

[2] J. L. Tonry et al., Astrophys. J. 594, (2003) 1; R. A. Knop et al., astro-ph/0309368; A. G. Riess et al., astro-ph/0402512.

[3] A. H. Jaffe et al., Phys. Rev. Lett. 86 (2001) 3475; A. E. Lange et al., Phys. Rev. D 63 (2001) 042001; A. Balbi et al., Astrophys. J. 545 (2000) L1; D. N. Spergel et al., astro-ph/0302209.

[4] S. Weinberg, Rev. Mod. Phys. 61 (1989) 1.

[5] C. Wetterich, Nucl. Phys. B 302 (1988) 668; B. Ratra and P. J. E. Peebles, Phys. Rev. D 37 (1988) 3406; R. R. Caldwell et al., Phys. Rev. Lett. 80 (1988) 1582; L. Wang et al., Astrophys. J. 530 (2000) 17; A. Albrecht and C. Skordis, Phys. Rev. Lett. 84 (2000) 2076; C. Armendariz-Picon, V. Mukhanov and P. J. Steinhardt, Phys. Rev. Lett. 85 (2000) 4438.

[6] T. Banks, hep-th/0007146; hep-th/0011255; T. Banks and W. Fischler, hep-th/0102077.

[7] S. Hellerman, N. Kaloper and L. Susskind, JHEP 0106 (2001) 003; W. Fischler, A. Kashani-Poor, R. McNees and S. Paban, JHEP 0107 (2001) 003.

[8] E. Witten, hep-ph/0002297.

[9] L. Dyson, M. Kleban and L. Susskind, JHEP 0210 (2002) 011; T. Banks, W. Fischler and S. Paban, JHEP 0212 (2002) 062; N. Goheer, M. Kleban and L. Susskind, JHEP 0307 (2003) 056.

[10] T. Banks and W. Fischler, astro-ph/0307459.

[11] D. A. Lowe and D. Marolf, hep-th/0402162.

[12] N. Kaloper, M. Kleban and L. Sorbo, Phys. Lett. B 600 (2004) 7.

[13] W. Fischler and L. Susskind, hep-th/9806039.

[14] R. Bousso, JHEP 9907 (1999) 004; JHEP 9906 (1999) 028; JHEP 0011 (2000) 038. 
[15] D. Bak and S. J. Rey, Class. Quant. Grav. 17 (2000) L83; R. Easther and D. A. Lowe, Phys. Rev. Lett. 82 (1999) 4967; G. Veneziano, Phys. Lett. B 454 (1999) 22; N. Kaloper and A. D. Linde, Phys. Rev. D 60 (1999) 103509; R. Brustein and G. Veneziano, Phys. Rev. Lett. 84 (2000) 5695.

[16] A. Guth, Phys. Rev. D 23 (1981) 347; A. Linde, Phys. Lett. B 108 (1982) 389; A. Albrecht and P. Steinhardt, Phys. Rev. Lett. 48 (1982) 1220.

[17] J. M. Bardeen, Phys. Rev. D 22 (1980) 1882.

[18] V. Mukhanov and G. Chibisov, JETP Lett. 33 (1981) 532; A. Starobinsky, Phys. Lett. B 117 (1982) 175; S. Hawking, Phys. Lett. B 115 (1982) 295; A. Guth and S.-Y. Pi, Phys. Rev. Lett. 49 (1982) 1110; J. Bardeen, P. Steinhardt and M. Turner, Phys. Rev. D 28 (1983) 679; A. Linde, Phys. Lett. B 116 (1982) 335; M. Sasaki, Prog. Theor. Phys. 70 (1983) 394; V. Mukhanov, JETP Lett. 41 (1985) 493.

[19] E. H. Gudmundsson and G. Bjornsson, Astrophys. J. 565 (2002) 1.

[20] A. Loeb, Phys. Rev. D 65 (2002) 047301; K. Nagamine and A. Loeb, New Astron. 8 (2003) 439; astro-ph/0310505.

[21] M. T. Busha, F. C. Adams, R. H. Wechsler and A. E. Evrard, Astrophys. J. 596 (2003) 713; F. C. Adams, M. T. Busha, A. E. Evrard and R. H. Wechsler, Int. J. Mod. Phys. D 12 (2003) 1743.

[22] G. W. Gibbons and S. W. Hawking, Phys. Rev. D 15 (1977) 2738. 